\title{
Riesgo Cardiovascular y Guías de Práctica Clínica: ¿aclaran o confunden? Repercusión según las diferentes Guías de Práctica Clínica utilizadas
}

\author{
Inmaculada Adán Valero ${ }^{a}$, Yoana Martínez Soriano ${ }^{\text {b }}$ \\ Javier Perona Caro ${ }^{c}$, David García Mateos ${ }^{d}$
}

\begin{abstract}
a Servicio de Urgencias del Hospital Gutiérrez-Ortega, Valdepeñas (Ciudad Real).

\author{
b Centro de Salud de \\ Honrubia, Honrubia (Cuenca).
} c Centro de Salud Tomelloso I,
Tomelloso (Ciudad Real).

a Servicio de Urgencias del Hospital Virgen de la Luz, Cuenca.

\section{Correspondencia:} Inmaculada Adán Valero, Servicio de urgencias, Hospital Gutiérrez-Ortega, Av. Estudiantes s/n, 13300 - Valdepeñas (Ciudad Real, España). Telf.: 645224574, e-mail: Inmaculadaadan@hotmail.com.

Recibido el 23 de febrero de 2009.

Aceptado para su publicación el 28 de mayo de 2009.

\section{RESUMEN}

Objetivo. Valorar la indicación de tratamiento antihipertensivo e hipolipemiante en prevención primaria, según las recomendaciones de las guías de práctica clínica más usadas en nuestro país.

Diseño. Estudio descriptivo transversal.

Emplazamiento. Centros de salud urbanos de Cuenca capital.

Participantes. Un total de 449 pacientes, entre 35 y 74 años, pertenecientes a los listados de hipertensos, diabéticos, dislipémicos, obesos y/o fumadores, en prevención primaria, de cuatro cupos pertenecientes a tres centros de salud de Cuenca capital.

Mediciones. Se calculó el riesgo cardiovascular (RCV) de cada paciente utilizando las tablas de Framingham, Regicor y Score. La correcta indicación de tratamiento fue valorada según los criterios del grupo PAPPS de la SEMFYC y CEIPC.

Resultados. El riesgo medio global fue: Framingham 11,31 \pm 8 (mediana 9), Regicor 4,63 $\pm 2,98$ (mediana 4), Score 4,06 $\pm 4,41$ (mediana 2). Según las recomendaciones PAPPS, un 39,9\% de los sujetos de la muestra deberían recibir tratamiento antihipertensivo, mientras que, siguiendo las recomendaciones Score-CEIPC, un 41,2\% debería recibir dicho tratamiento. El índice de concordancia simple fue del $91,1 \%$, con un índice Kappa de 0,82. Respecto al tratamiento hipolipemiante, según las recomendaciones PAPPS debería recibirlo un 8,5\%, frente a un $21,2 \%$ según las recomendaciones Score-CEIPC. El índice de concordancia simple fue del 82,4\%, con un índice Kappa de 0,32.

Conclusiones. Las dos guías más frecuentemente utilizadas en nuestro medio tienen una elevada concordancia a la hora de indicar tratamiento antihipertensivo, pero difieren bastante en la indicación de tratamiento hipolipemiante. Las recomendaciones de Score-CEIPC indican tratamiento hipolipemiante en una mayor proporción de pacientes que las recomendaciones del PAPPS.

Palabras clave. Modelos Cardiovasculares. Guías de Práctica Clínica. Agentes Antihipertensivos. Agentes Anticolesterémicos.

\section{ABSTRACT}

Cardiovascular risk and Clinical Practice Guidelines: clarification or confusion? Repercussion according to which Clinical Practice Guideline is used

Objective. To evaluate the indication of antihypertensive and lipid-lowering treatment in primary prevention, according to the recommendations of the most commonly used clinical practice guidelines in Spain.

Design. Descriptive Cross-sectional Study.

Setting. Urban Primary Health Care Centres in Cuenca.

Participants. A total of 449 individuals aged between 35 and 74 , recorded as having hypertension, hypercholesterolemia, diabetes mellitus, obesity or being smokers, without previous cardiovascular disease, corresponding to four GPs quotas at three healthcare centres in the city of Cuenca, Spain. Main measurements. CVR was determined using risk the equations of Framingham, REGICOR and SCORE. The appropriate use of treatment was assessed according to the criteria of the Program on Prevention and Health Promotion Activities for Primary Care (PAPPS), the Spanish Association for Family and Community Medicine (SEMFYC) and the Spanish Interdisciplinary Committee for the Prevention of Cardiovascular Disease (CEIPC).

Results. The average overall risk was: Framingham $11.31 \pm 8$ (median 9); Regicor $4.63 \pm 2.98$ (median 4); Score $4.06 \pm 4.41$ (median 2). According to the PAPPS recommendations, $39,9 \%$ of the subjects should receive antihypertensive treatment, whereas, according the SCORE-CEIPC recommendations, 41,2 \% should receive such treatment. (IC 91,1 \%), (Kappa 0,82). With regard to lipid-lowering treatment, according to the PAPPS recommendations $8.5 \%$ should receive treatment compared to $21.2 \%$ according to the SCORE-CEIPC recommendations. (IC 82,4\%), (Kappa 0,32).

Conclusions. The two most frequently used guidelines lines in Spain show high conformity for indicating antihypertensive treatment, but they differ greatly in the indication of lipid-lowering treatment. The SCORE-CEIPC recommendations indicate lipid lowering treatment in a greater percentage of patients than do those of PAPPS.

Key words. Models, Cardiovascular. Practice Guidelines. Antihypertensive Agents. Anticholesteremic Agents.

El presente estuydio forma parte de otro más extenso que recibió una beca para investigadores noveles por parte de la Fundación para Investigación Sanitaria en Castilla-La Mancha (FISCAM) y fue presentado parcialmente en el VII Congreso de Atención Primaria de Castilla La Mancha, celebrado en Cuenca en Junio de 2006. 


\section{INTRODUCCIÓN}

Las enfermedades cardiovasculares son la primera causa de muerte en España, representando el 33,72\% del total de defunciones ${ }^{1}$. Además, gran parte de estas muertes ocurren de manera súbita en pacientes asintomáticos, lo que indica un desarrollo subclínico de la enfermedad ${ }^{2}$.

Las tasas ajustadas de mortalidad por enfermedades cardiovasculares han disminuido en España desde 1975. En el periodo 1985-1995 se redujeron un $26 \%$, con una disminución media anual del $2,4 \%{ }^{3}$. A pesar de esta disminución, debido al envejecimiento poblacional y al aumento de la supervivencia, el impacto sobre la salud medido por el número de enfermos y el uso de servicios sanitarios sigue siendo muy alto. Este impacto se incrementará a lo largo de las próximas décadas, generando una elevada demanda asistencial. Según estimaciones, en el año 2020 la cardiopatía isquémica seguirá siendo la primera causa de muerte en los países industrializados y la tercera en los países en vías de desarrollo ${ }^{4,6,16}$.

La solución pasa por hacer un mayor énfasis en la prevención de la enfermedad, es decir, no esperar a la aparición de síntomas sino actuar precozmente frente a los factores de riesgo, lo que constituye el concepto de disminución del riesgo ${ }^{5}$.

La estimación de que un individuo desarrolle un acontecimiento cardiovascular a partir de sus factores de riesgo constituye un elemento valioso para la prevención primaria, estando ampliamente reconocido que una evaluación del riesgo global de un paciente permite tomar decisiones más eficientes que mediante el abordaje de los factores de riesgo por separado ${ }^{7}$. De este concepto surge la idea de englobar los principales factores de riesgo cardiovasculares en tablas que estratifiquen el riesgo global de los pacientes de un modo más eficiente.

En las últimas décadas se han publicado distintas tablas que calculan el riesgo cardiovascular global. Hasta ahora las más utilizadas eran las basadas en el estudio de Framingham ${ }^{8}$. En el año 2003 se publicaron las tablas basadas en el estudio REGICOR ${ }^{6}$, sustentadas en los registros de cardiopatía isquémica de la provincia de Gerona y calibradas según la ecuación de Framingham clásica. Recientemente se han publicado las tablas SCORE que derivan de una gran base de datos de estudios prospectivos de diferentes países europeos, imponiéndose poco a poco en la práctica clínica diaria9. En la actualidad es el recomendado por la Guía Europea de prevención cardiovascular en la práctica clínica (Third Joint Task Force Europeo 2003) para el tratamiento de la HTA y la hipercolesterolemia.
Vemos así que la intervención farmacológica en prevención primaria de la enfermedad cardiovascular ha sido un tema controvertido y abordado desde diferentes criterios a lo largo de los últimos años ${ }^{6,18}$. La variabilidad de la práctica clínica en la prescripción de fármacos hipolipemiantes y antihipertensivos ha estado motivada por los diversos criterios de actuación aportados por las distintas sociedades científicas, así como por la utilización de diferentes versiones de la ecuación de Framingham para el cálculo cuantitativo del riesgo cardiovascular $19,20,21,22$. De la necesidad de adecuar el tratamiento antihipertensivo e hipolipemiante de los pacientes, según el riesgo cardiovascular estimado con las diferentes tablas de estratificación, surgen las guías de práctica clínica, entre las que destacan la guía PAPPS y la actualmente promulgada por las Sociedades Europeas.

La Guía de Prevención Cardiovascular PAPPS ${ }^{13}$ es una herramienta para tomar decisiones clínicas según el riesgo cardiovascular estimado de los pacientes y hasta la actualidad ha sido de referencia a nivel nacional, basándose en la ecuación de Framingham y la de REGICOR a la hora de adecuar el tratamiento farmacológico a los pacientes.

En la Guía Europea para la prevención de enfermedades cardiovasculare ${ }^{14}$ han participado conjuntamente varias sociedades científicas europeas. El Comité Español Interdisciplinario para la Prevención Cardiovascular (CEIPC) ha traducido y adaptado dicha guía con el consenso de la administración sanitaria y las 11 sociedades científicas españolas involucradas en prevención cardiovascular. En esta guía y en el consenso suscitado desde su publicación se basa la tabla SCORE para dictaminar el tratamiento para los pacientes según el riesgo cardiovascular previamente medido.

La existencia de múltiples tablas para calcular el riesgo cardiovascular y varias guías de práctica clínica, de cuyo uso se derivan diferentes actuaciones terapéuticas, crea bastante confusión entre los profesionales. Así, en diferentes estudios se ha puesto de manifiesto cómo se modifican no sólo los niveles de riesgo de los pacientes sino también la indicación de tratamiento según las diferentes tablas y guías utilizadas ${ }^{15}$.

Lo ideal sería disponer de una tabla de cálculo del RCV basada enteramente en datos de toda la población española y de una guía de práctica clínica elaborada por las sociedades científicas españolas y aceptadas por todas las administraciones con responsabilidades sanitarias.

La aparición de nuevas tablas adaptadas a nuestro medio podría disminuir la sobreestimación que la formulación de Framingham origina en el cálculo del 
riesgo cardiovascular global en la población española, y esto podría reducir la sobre-utilización de medidas farmacológicas. Sin embargo, la ausencia de una tabla y una guía de esas características nos llevan a proponer el presente estudio, que pretende arrojar algo de luz sobre esta situación imprecisa, ayudando al profesional a decantarse por una tabla determina$\mathrm{da}$, tras conocer las ventajas, los detrimentos y las limitaciones de cada una de ellas.

El objetivo principal de este trabajo ha sido determinar la indicación de tratamiento antihipertensivo e hipolipemiante, en prevención primaria, según distintos cálculos del riesgo cardiovascular global y las recomendaciones de iniciar tratamiento de las distintas sociedades científicas.

\section{MATERIAL Y MÉTODO}

Se realizó un estudio descriptivo transversal que incluyó a 449 pacientes pertenecientes a los listados de hipertensos, diabéticos, dislipémicos, obesos y/o fumadores en prevención primaria de 4 cupos de los tres centros de salud de Cuenca capital y con edades comprendidas entre 35 y 74 años.

Se excluyó a los pacientes menores de 35 y a los mayores de 74 años, así como a aquellos con antecedentes de eventos cardiovasculares, cerebrovasculares o arteriopatía periférica. Tampoco se incluyó a los pacientes que tras ser informados de dicho estudio no quisieron participar en él.

Se utilizó un muestreo sistemático con arranque aleatorio a partir del listado de sujetos de 35 a 74 años, ambos inclusive, de los 4 cupos anteriormente citados. Los pacientes fueron incluidos entre septiembre de 2005 y junio de 2006.

El tamaño muestral fue estimado a partir de la diferencia de proporciones de pacientes de alto riesgo en cada tabla, asumiendo una diferencia estimada del $10 \%$, una precisión de $\pm 3 \%$, un nivel de confianza del $95 \%$ y una potencia del $90 \%$, resultando un tamaño de 319 pacientes. Asumiendo unas pérdidas del $20 \%$, el número total de pacientes estimado fue de al menos 400 .

Se contactó telefónicamente con los pacientes, realizándose una primera llamada en horario de tarde; en el caso de no contactar con el paciente se repitió la llamada otro día en horario de mañana. Realizamos 4 intentos antes de desestimar al paciente. En caso de no poder contactar con el paciente, se llamó al inmediatamente posterior en el listado. Si el paciente no acudía a la entrevista o se negaba a acudir, se le consideró no respondedor, y se recogieron sus variables sociodemográficas para valorar si existían diferencias estadísticamente significativas entre respondedores y no respondedores. Previamente a la inclusión de cada paciente en el estudio, se solicitó su consentimiento informado por escrito.

Las variables que se recogieron fueron: variables sociodemográficas de importancia para valorar las características de nuestra población (estado civil, nivel educativo, situación laboral, etc.), variables necesarias para realizar el cálculo del riesgo cardiovascular con las distintas tablas (presión arterial sistólica y diastólica, peso, talla, índice de masa corporal -IMC-, glucemia basal, niveles de triglicéridos, colesterol total, c-HDL, c-LDL, creatinina, tras al menos 10 horas de ayuno, habito tabáquico, sexo, edad y presencia no de hipertrofia de ventrículo izquierdo), riesgo cardiovascular, medido con las tablas de Framingham, REGICOR y SCORE, y recomendaciones respecto a la prescripción de antihipertensivos e hipolipemiantes con la guía PAPPS y la de Sociedades Europeas.

Los datos, incluidos en la base de datos, fueron sometidos a un análisis con el programa estadístico SPSS. La descripción de la muestra se realizó de forma global y por sexos, utilizando para las variables continuas la media y la desviación estándar y para las categóricas los porcentajes. También se describió de forma similar a los pacientes clasificados de riesgo alto por cada una de las tres ecuaciones: Framingham, REGICOR y SCORE. Otros datos que se obtuvieron fueron el riesgo medio y el porcentaje de pacientes de riesgo alto según dichas ecuaciones. La concordancia en la clasificación de pacientes de riesgo alto se analizó mediante el porcentaje de concordancia global y de cada grupo y el índice Kappa. Las diferencias entre las variables de estudio se analizaron mediante los intervalos de confianza del 95\%. Se realizó un análisis bivariante utilizando la t de Student para diferencia de medias y la chi cuadrado para diferencia de proporciones. Debido a las nuevas recomendaciones REGICOR (alto riesgo cardiovascular cuando la probabilidad de evento vascular es mayor o igual al 10\%), se analizaron ambos puntos de corte (REG. $\geq 20 \%$ y REG. Modificado $\geq 10 \%$ ).

\section{RESULTADOS}

La población final de estudio estuvo constituida por 449 pacientes. La edad media de los participantes fue de $60,5 \pm 10,34$ años, con una proporción de mujeres del $60,1 \%$ y de hombres del $39,9 \%$. Eran fumadores un $19,6 \%$, exfumadores un $19,6 \%$ y diabéticos un $21,6 \%$. La cifra media de TAS fue $139,03 \pm 18,98$, de TAD 84,29 $\pm 11,50$, de colesterol total $213,27 \pm 35,74$ y de IMC 30,25 $\pm 5,19$.

El riesgo medio global obtenido utilizando la tabla de Framingham clásica fue de 11,31 \pm 8 (mediana 9), con 


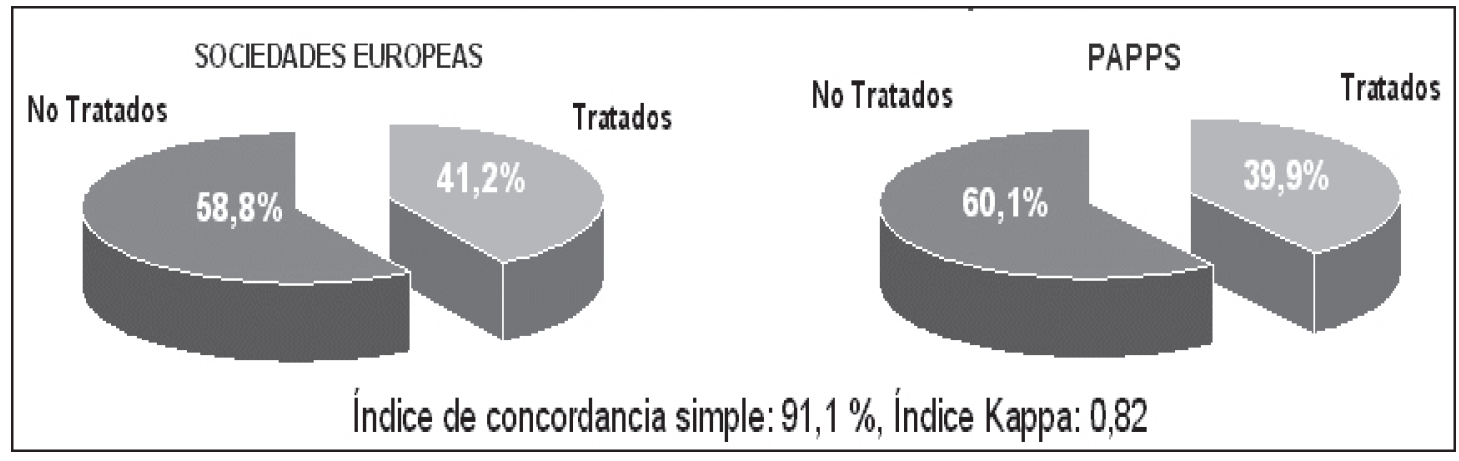

Figura 1. Recomendaciones de tratamiento antihipertensivo.

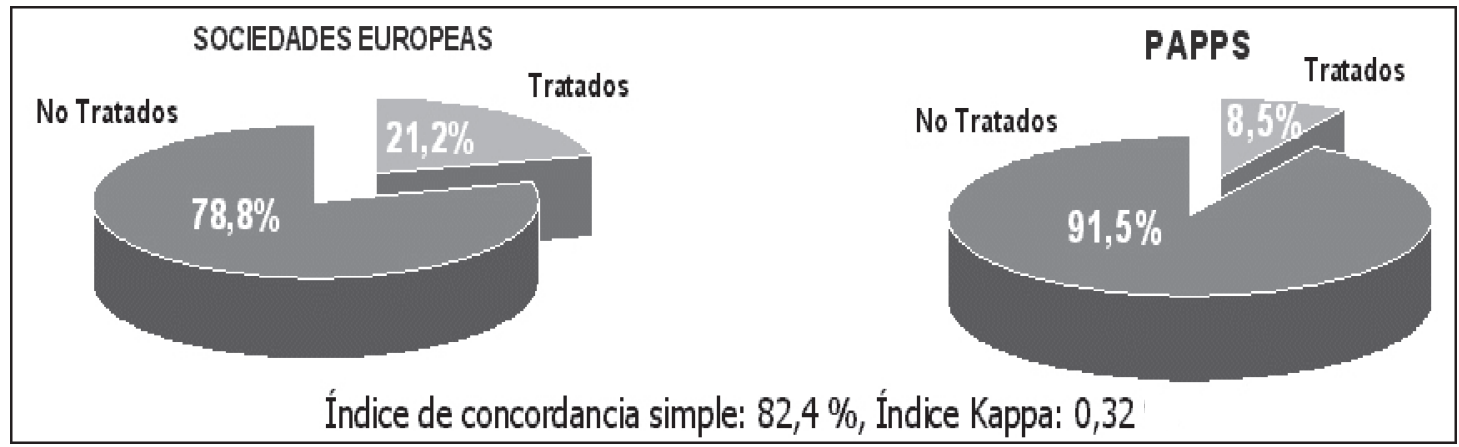

Figura 2. Recomendaciones de tratamiento hipolipemiante.

REGICOR de 4,63 $\pm 2,98$ (mediana 4 ) y con SCORE de 4,06 $\pm 4,41$ (mediana 2).

Según las recomendaciones del PAPPS, se debería tratar con fármacos antihipertensivos un $39,9 \%$ de los sujetos de la muestra, mientras que según las recomendaciones de las sociedades europeas un $41,2 \%$ debería recibir dicho tratamiento. El índice de concordancia simple fue del $91,1 \%$, con un índice kappa de 0,82 (figura 1).

Al aplicar las recomendaciones de tratamiento antihipertensivo del PAPPS al riesgo calculado con la tabla REGICOR modificado, comprobamos que un $37 \%$ de nuestra muestra debería recibir tratamiento antihipertensivo, frente al 33,2\% que debería recibirlo al aplicar Regidor clásico. Estos resultados (REGICOR modificado) suponen un índice de concordancia simple con las recomendaciones de las Sociedades Europeas del 90,8\%, con un índice Kappa del 0,81.

Respecto a la necesidad de instauración de tratamiento hipolipemiante, según las recomendaciones del PAPPS debería recibirlos un 8,5\%, frente a un $21,2 \%$ según las recomendaciones de las Sociedades Europeas. El índice de concordancia simple fue del 82,4\%, con un índice kappa del 0,32 (figura 2).
Hay un $15,1 \%$ de pacientes en los que las sociedades europeas indican tratamiento hipolipemiante, pero el PAPPS no lo hace, y un $2,4 \%$ en los que ocurre lo contrario. Según la guía PAPPS, la indicación de tratamiento hipolipemiante en nuestros pacientes, tras haberles calculado el riesgo cardiovascular con la tabla REGICOR modificada, pasaría a ser de un 5,6\%, frente al $3,3 \%$ con REGICOR sin modificar, lo que supone un índice de concordancia simple con la indicación de las Sociedades Europeas de un 80,9\%, con un índice kappa del 0,20. Si comparamos las indicaciones de tratamiento hipolipemiante de la guía PAPPS, al utilizar Framingham clásica, con REGICOR Modificado, obtenemos un índice de concordancia simple del $96,2 \%$, con un kappa del 0,17.

\section{DISCUSIÓN}

En este estudio vemos una clara variabilidad en el resultado final de la valoración de los pacientes según la tabla empleada para el cálculo del riesgo cardiovascular y la aplicación de los distintos criterios de intervención establecidos por el grupo PAPPS y las Sociedades Europeas. Ante esto, habría que tener en cuenta que las tablas de Framingham y REGICOR estiman el riesgo coronario, mientras que la tabla SCO$\mathrm{RE}$ calcula el riesgo de mortalidad cardiovascular. 
Obtenemos una buena correlación entre la ecuación de Framingham y REGICOR modificada, lo cual era esperable si se tiene en cuenta que REGICOR ha sido calibrada a partir de Framingham y sobre la base de una metodología ya validada en otras poblaciones ${ }^{6,23}$. La correlación fue inferior al comparar las tablas Framingham y REGICOR con SCORE. Esto era esperable al tratarse de tablas con una formulación distinta.

Si analizamos los resultados obtenidos en este estudio, podemos ver que el riesgo medio global obtenido es algo superior al de la población general. Esto puede deberse a que para la realización del mismo se seleccionó a pacientes incluidos en programas de riesgo cardiovascular en prevención primaria, y por tanto ya presentaban algún factor de riesgo cardiovascular. Este hecho supone una limitación a la hora de extrapolar los resultados de este estudio a la población general.

Además, para la realización de este estudio se han utilizado cupos urbanos, no disponiendo de datos sobre la variación del riesgo cardiovascular en personas que viven en el medio rural. Vivir en el medio rural podría influir en el cálculo del riesgo cardiovascular, ya que variables como el sedentarismo o la alimentación pueden influir en las cifras de tensión arterial o de colesterol, modificando así el riesgo cardiovascular.

Al analizar los resultados obtenidos comprobamos que la edad media de los sujetos de nuestra muestra fue bastante elevada (60,5 $\pm 10,34$ años), lo cual se debe a dos motivos principalmente: por un lado, a que la mayoría de los pacientes incluidos en programas de prevención primaria son mayores de 40 años, y, por otro lado, a que los pacientes menores de 40 años rehusaron participar en el estudio alegando falta de tiempo o desinterés, al no considerar muchos de ellos que el tabaquismo, principal motivo por el que este grupo se encontraba en programas de prevención, se relacionase con aumentos en su riesgo cardiovascular.

En cuanto a la distribución por sexo, la proporción de mujeres que participó en el estudio $(60,1 \%)$ fue muy superior a la de los varones. Esto puede deberse a factores tanto socioculturales como clínico-patológicos. Por un lado, se observó que las mujeres de estos cupos tenían una mayor concienciación en cuanto a prevención y hábitos saludables, siendo más accesibles a la hora de participar en el estudio. Por otro lado, también es importante reseñar que, debido a la mayor incidencia de eventos cardiovasculares en varones en nuestro medio, muchos debieron ser excluidos por considerarse ya en prevención secundaria.

En cuanto a las tasas medias de TAS, TAD y CT, en esta muestra son algo más bajas que en la población general, lo cual puede ser debido a que muchos de los sujetos incluidos recibían tratamiento antihipertensivo y/o hipolipemiante, siendo éste otro motivo para que estos datos no se puedan extrapolar a la población general.

Cuando se analiza la indicación de tratamiento antihipertensivo en la muestra, se obtiene que un 39,9\% debería seguir tratamiento con las recomendaciones Framingham-PAPPS, frente al $41,2 \%$ con las recomendaciones SCORE-CEIPC (índice de concordancia simple 91,1\%; índice Kappa 0,82). Si analizamos la indicación REGICOR modificado ( $\geq 10 \%)$-PAPPS, un $37 \%$ de nuestra muestra debería recibir tratamiento antihipertensivo. Estos datos son muy similares a los encontrados con las recomendaciones Framingham-PAPPS, Presentando un índice de concordancia simple con las recomendaciones de las Sociedades Europeas (SCORE-CEIPC) del 90,8\%, (índice Kappa 0,81 ). A pesar de la elevada concordancia, hay una mayor proporción de pacientes que serían tratados con las recomendaciones SCORE-CEIPC.

Respecto al tratamiento hipolipemiante según las recomendaciones Framingham-PAPPS, debería recibir tratamiento un $8,5 \%$ de pacientes, frente al $21,2 \%$ que lo recibiría según las recomendaciones de las Sociedades Europeas (Índice de concordancia simple 82,4\%; Kappa 0,32). La mayor indicación de tratamiento con las recomendaciones de las Sociedades Europeas (SCORE-CEIPC), puede deberse a la introducción en la muestra de los pacientes diabéticos, que suponían un $21,6 \%$.

Con el presente estudio vemos como la elección de una determinada tabla, así como la recomendación de tratamiento que lleve asociada, puede tener importantes repercusiones en la práctica clínica, y traducirse en distintas intervenciones terapéuticas. Dada la alta prevalencia de hipertensión arterial e hipercolesterolemia en nuestro medio, las implicaciones económicas derivadas tanto del sobretratamiento como de no tratar correctamente merecen atención. Por eso, la elección de la tabla y guía a usar debería justificarse según refleje con mayor exactitud el riesgo cardiovascular de la población a la que se aplica. Además, los pacientes candidatos a ser tratados deben ser aquellos de los que se disponga de una mayor evidencia científica.

Como conclusión, las dos guías más frecuentemente utilizadas en nuestro medio tienen una elevada concordancia a la hora de indicar el tratamiento antihipertensivo (un $39,9 \%$ frente a un $41,2 \%$ ). Sin embargo, existe discordancia entre ambas guías a la hora de indicar tratamiento hipolipemiante (un $8,5 \%$ respecto a un $21,2 \%)$. Las recomendaciones de las sociedades 
europeas indican tratamiento hipolipemiante en una mayor proporción de pacientes que las recomendaciones del PAPPS.

\section{AGRADECIMIENTOS}

Nuestro más sincero agradecimiento a FISCAM por la confianza depositada en nosotros, así como a la Unidad Docente de Medicina de Familia de Cuenca y a los tutores de los centros de salud, muy en especial a $D^{\mathrm{a}} \mathrm{M}^{\mathrm{a}}$ de los Ángeles Gabriel Escribano y a $\mathrm{D}^{\mathrm{a}} \mathrm{Sa}-$ grario Sáiz Santos por prestarnos su colaboración en este proyecto.

\section{BIBLIOGRAFÍA}

1. Instituto Nacional de Estadística. Indicadores Demográficos Básicos. Mortalidad. Disponible en: www.ine.es

2. Marrugat J, Elosua R, Martí H. Epidemiología de la cardiopatía isquémica en España: estimación del número de casos y de las tendencias entre 1997 y 2005. Rev Esp Cardiol 2002; 55:33748.

3. Villar Álvarez F, Banegas Banegas JR. Reducir las enfermedades cardiovasculares. En: Álvarez Dardet C, Peiró S (eds). La Salud Pública ante los desafíos de un nuevo siglo. Informe SESPAS 2000. Madrid: SESPAS; 2000.

4. Murria CL, Lopez A. Alternative projections of mortality and disability by cause. 1990-2020: global burden of disease study. Lancet 1997; 549:1498-504.

5. Fuster V, Gotto M. Risk reduction. Circulation 2000; 102:94102.

6. Marrugat J, Solanas P, D‘Agostino R, Sullivan L, Ordovás J, Cordón $F$, et al. Estimación del riesgo coronario en España mediante la función de Framingham calibrada. Rey Esp Cardiol 2003; 56(3);253-61.

7. Prevention of coronary Herat disease in clinical practice. Recommendations of the second joint task force of European and the other societies on coronary prevention. Eur Heart J 1998; 19:1434-503.

8. Kannel WB, Castelli WP, Gordon T, McNamara PM. Serum cholesterol, lipoproteins, and the risk of coronary heart disease. The Framminghan study. Ann Intern Med 1971; 74:1-12.

9. Conray RM, Pyorala K, Fitzgeral AP, Sans S, Menotti A, De Backer $\mathrm{G}$, et al. Estimation of ten year risk of fatal cardiovascular disease in Europe: the SCORE Project. Eur Heart J 2003; 24:987-1003.

10. Álvarez Cosmea A. Las tablas de riesgo cardiovascular. Una revisión crítica. Medifam 2001; 11:122-39.
11. Anderson KM, Wilson PWF, Odell PM, Kannel WB. An Updated coronary risk profile. A Statement for health profesionais. Circulation 1991; 83:356-62.

12. Marrugat J, Fiol M, Sala J, Tormo Mi, Segura A, Muñiz V, et al. Variabilidad geográfica en España en las tasas de incidencia y mortalidad poblacionales por infarto agudo en el estudio IBERIA. Málaga. Rey Esp Cardiol. 2000; 53(supl 2):71.

13. Grupo de Prevención Cardiovascular del Programa de Actividades Preventivas y Promoción de la Salud de la Sociedad Española de Medicina de Familia y Comunitaria (SEMFyC). Guía de prevención cardiovascular. Madrid: Sersa; 1996.

14. Backer G, Ambrosioni E, Borch-Johnsen K, Brotons C, Cifkova $R$, Dallongeville J et al. Guía Europea de Prevención Cardiovascular en la práctica clínica. Aten Primaria 2004; 34(8):427-32.

15. Ramos R, Solanas P, Cordon F, Rohlfs I. Elosva R, Sala J. et al. Comparación de la función de Framingham original y la calibrada del REGICOR en la predicción del riesgo coronario poblacional. Med Clin (Barc) 2003; 121(14);521-6.

16. Murria CL, Lopez A. Alternative projections of mortality and disbility by cause 1990-2020: Global Burden of Disease Study. Lancet 1997; 349:1498-504.

17. Maiques Galan A, Antón Garcia F, Franch Taix M, Albert Ros X, Aleixandre Martí E y Collado Gil A. Riesgo cardiovascular del SCORE comparado con el de Framingham. Consecuencias del cambio propuesto por las Sociedades Europeas. Med Clin (Barc) 2004; 123(18):681-5.

18. Downs G, Clearfield M, Weiss S, Whitney E, Shapiro D, Beere $P$, et al. Prevención primaria de los eventos coronarios agudos con lovastatina en varones y mujeres con concentraciones promedio de colesterol. Resultados del AFCAPS/TexCAPS. JAMA (ed. esp.) 1998; 7:305-313.

19. Plaza Pérez I, Villar Alvárez F, Mata López P, Pérez Jiménez F, Maiques Galán A, Casanovas Lenguas JA, et al. Control de la colesterolemia en España, 2000. Un instrumento para la prevención cardiovascular. Disponible en: http//www.searteriosclerosis.org/consenso.htm.

20. Primary prevention of coronary heart disease: guidance from Framingham. Circulation 1998; 97:1876-1887.

21. Villar Alvárez F, Galán Maiques C, Brotons Cuixart C, Torcal Laguna J, Lorenzo Piqueras A, Vilaseca Canals J, et al. Prevención Cardiovascular en atención primaria. Aten Primaria 2001; 28 Supl 2:13-26

22. Pérez G, Pena A, Sala J, Roset PN, Masiá R, Marrugat $J$ and the REGICOR Investigators. Acute myocardial infarction case fatality, incidence and mortality rates in a population registry in Girona, Spain, 1990-92. Int J Epidemiol 1998; 27:599-604.

23. Baena JM, Del Val JL, Salas LH, Sánchez R, Altes E, Deixens $B$ et al. Comparación de los modelos SCORE y REGICOR para el cálculo del riesgo cardiovascular en sujetos sin enfermedad cardiovascular atendidos en un centro de salud de Barcelona. Rev Esp Salud Púb 2005; 79:453-464. 